\title{
ALTERNATIVAS ANALÍtiCAS PARA DETERMINAÇÃo DE FERRO E TITÂNIO EM CIMENTO PORTLAND
}

Jorge de O. Franco Jr., Maria das Graças A. Korn *, Antonio Celso S. Costa e Anibal de Freitas Santos Jr. Departamento de Química Analítica, Universidade Federal da Bahia, 40170-290 Salvador - BA

Leonardo S. G. Teixeira

Departamento de Química Analítica, Universidade Federal da Bahia, 40170-290 Salvador - BA

Departamento de Engenharia, Universidade Salvador, UNIFACS, Av. Cardeal da Silva, 747, 40220-141 Salvador - BA

Recebido em 19/04/00; aceito em 1/9/00

\begin{abstract}
COMPARATIVE STUDY OF ANALYTICAL METHODS FOR IRON AND TITANIUM DETERMINATION IN PORTLAND CEMENT. In the present work four different analytical methodologies were studied for the determination of iron and titanium in Portland cement. The cement samples were dissolved with hot $\mathrm{HCl}$ and $\mathrm{HF}$, being compared $\mathrm{Fe}$ and $\mathrm{Ti}$ concentrations through four analytical methods: molecular absorption spectrophotometry using the reagents 1,2-hydroxybenzene-3,5-disulfonic acid (Tiron) and the 5-chloro-salicylic acid (CSA), inductively coupled plasma atomic emission spectrometry (ICP-AES) and flame atomic absorption spectrophotometry (FAAS). In the spectrophotometric determinations were studied $\mathrm{pH}$ conditions, reagents addition order, interferences, amount of reagents, linear range and stability of the system. In the techniques of ICP-AES and FAAS were studied the best lines, interferences, sensibility and linear range. The obtained results were compared and the agreement was evaluated among the methods for the determination of the metals of interest.
\end{abstract}

Keywords: Portland cement; iron determination; titanium determination.

\section{INTRODUÇÃO}

Para avaliarmos a importância do cimento na sociedade moderna basta tentar imaginar como seria nossa civilização sem túneis, pontes, viadutos, represas, etc. A resistência, versatilidade e durabilidade desta classe de material justificam a sua aplicabilidade, movimentando bilhões de dólares anualmente em todo o mundo. Com o objetivo de controlar sua qualidade e evitar a poluição do ambiente com metais tóxicos, que podem estar presentes em sua composição, o cimento está entre as matrizes que devem ser analisadas rotineiramente ${ }^{1}$.

$\mathrm{O}$ processo de preparo do cimento depende do controle das proporções e da granulometria das matérias-primas envolvidas para que um contato íntimo entre elas permita que as conversões químicas desejadas ocorram na temperatura de calcinação no forno. No processo de fabricação do cimento ocorrem diversas reações que serão responsáveis por suas características finais. Estas transformações originam diversos compostos. As funções diferenciadas de cada componente do cimento Portland induzem a necessidade de métodos de análise química deste material, uma vez que suas características irão depender da sua composição.

O monitoramento dos níveis de concentração de ferro e titânio no cimento é de fundamental importância. O titânio e outros componentes não voláteis do cimento atuam decisivamente no processo de cozimento. Variações na concentração de titânio podem modificar a viscosidade e a tensão superficial, o tamanho dos grãos, o rendimento e a resistência do cimento. Variações na concentração de ferro podem afetar a resistência do cimento além de alterar a temperatura de formação do clínquer².

Existem vários reagentes orgânicos que são utilizados para determinações espectrofotométricas de ferro e titânio. Entretanto, são poucos que permitem a determinação simultânea desses dois metais. Dentre os reagentes espectrofotométricos usados para determinação simultânea de Fe e Ti pode-se destacar o ácido 1,2-dihidroxibenzeno-3,5-dissulfônico (Tiron) ) $^{3,4}$ e o ácido 5-cloro salicílico $(\mathrm{CSA})^{2}$ devido à boa sensibilidade,

*E-mail: korn@ufba.br seletividade e estabilidade tanto do reagente como dos complexos formados.

Técnicas de espectrometria atômica tais como a espectrometria de emissão atômica com plasma de argônio indutivamente acoplado (ICP-AES) ${ }^{5,6}$ e espectrometria de absorção atômica com atomização por chama (FAAS) ${ }^{7-9}$ também são utilizadas para determinação de ferro e titânio em cimentos. As determinações com ICP-AES ou FAAS apresentam uma série de vantagens, tais como: rapidez, fácil correção dos efeitos de matriz e interferências espectrais, uma ampla faixa linear, excelente reprodutibilidade e grande versatilidade ${ }^{10-11}$

No presente trabalho, foram desenvolvidas e comparadas quatro metodologias analíticas para a determinação de ferro e titânio em cimento Portland: espectrofotometria de absorção molecular utilizando Tiron e CSA, espectrometria de emissão atômica com plasma indutivamente acoplado e espectrometria de absorção atômica com chama. Nas determinações espectrofotométricas foram estudadas as condições de $\mathrm{pH}$, ordem de adição de reagentes, presença de interferentes, quantidade de reagente e tampão, faixa linear e estabilidade. Nas técnicas de ICP-AES e FAAS foram avaliadas as melhores linhas, presença de interferência, sensibilidade e faixa de trabalho. Os resultados obtidos com as quatro metodologias foram comparados e avaliou-se a concordância entre os métodos para a determinação dos metais de interesse.

\section{PARTE EXPERIMENTAL}

\section{Reagentes}

Todos os reagentes usados foram de grau analítico e as soluções preparadas com água destilada-desionizada.

Soluções padrão de ferro $1,79 \times 10^{-2} \mathrm{~mol} \mathrm{~L}^{-1}$ e solução padrão de titânio $2,09 \times 10^{-2} \mathrm{~mol} \mathrm{~L}^{-1}$. Foram preparadas partindo de soluções contidas em ampolas Titrisol (Merck). As soluções de trabalho foram preparadas através de diluições apropriadas.

Solução tampão ácido acético/acetato de sódio $1,0 \mathrm{~mol} \mathrm{~L}^{-1}$ ( $p H 4,75)$. Foi preparada ajustando-se uma solução de acetato de sódio (Merck) 1,0 mol L-1 com solução de ácido acético (Merck) 1,0 mol L-1 para o $\mathrm{pH}$ desejado. 
Solução de ácido 1,2-dihidroxibenzeno-3,5-dissulfônico (Tiron) $3,01 \times 10^{-2} \mathrm{~mol} \mathrm{~L}^{-1}$. Dissolveu-se 1,0 g de Tiron (Aldrich) com água desionizada e o volume foi completado para $100 \mathrm{~mL}$.

Solução de ácido 5-cloro salicílico (CSA) $1,45 \times 10^{-1} \mathrm{~mol} \mathrm{~L}^{-1}$. Dissolveu-se 2,5 g de CSA (Merck) em $40 \mathrm{~mL}$ de álcool etílico e adicionou-se $60 \mathrm{~mL}$ de Brij-35 $10 \%(\mathrm{~m} / \mathrm{v})$.

Solução de Brij-35 10\% (m/v). Dissolveu-se $10 \mathrm{~g}$ de Brij35 (Merck) com água desionizada e o volume foi completado para $100 \mathrm{~mL}$.

\section{Instrumental}

As medidas de absorvância foram obtidas empregando espectrofotômetro UV-visível duplo-feixe Varian modelo Cary $1 \mathrm{E}$, utilizando celas de $10 \mathrm{~mm}$ de caminho óptico. Os ajustes de $\mathrm{pH}$ foram feitos em potenciômetro Digimed modelo DM 20 (com eletrodo de vidro combinado DME-MV1). Para os estudos com ICP-AES foi empregado um Espectrômetro de Emissão Atômica com Plasma Indutivamente Acoplado, Fisons ARL 3410 , equipado com minitocha. Para as determinações com FAAS empregou-se um Espectrômetro de Absorção Atômica Varian modelo 220. As condições de operação do ICP-AES e do FAAS são mostradas nas Tabelas 1 e 2 respectivamente.

Tabela 1. Condições de operação do Espectrômetro de Emissão Atômica com Plasma de Argônio Induzido (ARL 3410)

\begin{tabular}{ll}
\hline Parâmetro & Condição operacional \\
\hline Potência Refletida & $<10 \mathrm{~W}$ \\
Potência Incidente & $650 \mathrm{~W}$ \\
Vazão do gás de Arraste & $0,8 \mathrm{~L} \mathrm{~min}{ }^{-1}$ \\
Vazão do Gás Refrigerante & $7,4 \mathrm{~L} \mathrm{~min}-1$ \\
Vazão do Gás Auxiliar & $0,8 \mathrm{~L} \mathrm{~min}{ }^{-1}$ \\
Vazão da Amostra & $2,30 \mathrm{~mL} \mathrm{~min}^{-1}$ \\
Comprimento de Onda & $\mathrm{Fe}-259,94 \mathrm{~nm}$ \\
& $\mathrm{Ti}-334,94 \mathrm{~nm}$ \\
\hline
\end{tabular}

Tabela 2. Condições operacionais do Espectrofotômetro de Absorção Atômica (Varian 220)

\begin{tabular}{ll}
\hline Parâmetro & Condição operacional \\
\hline Tipo de lâmpada & cátodo ôco \\
Corrente da lâmpada & $5 \mathrm{~mA}$ \\
Combustível & Acetileno \\
Suporte / Comburente & $\mathrm{Ar}(\mathrm{Fe})$ e Óxido Nitroso (Ti) \\
Largura da fenda & $0,1 \mathrm{~nm}$ \\
Estequiometria da chama & Oxidante $(\mathrm{Fe})$ e Redutora (Ti) \\
Comprimento de Onda & $\mathrm{Fe}-248,3 \mathrm{~nm}$ \\
& $\mathrm{Ti}-364,3 \mathrm{~nm}$ \\
\hline
\end{tabular}

\section{Preparação das Amostras}

Pesou-se exatamente porções entre 0,2500 e 0,250 g de cada amostra de cimento em béquer de PTFE. Adicionou-se $25 \mathrm{~mL}$ de $\mathrm{HCl}_{\text {conc. }}$ e $10 \mathrm{~mL}$ de $\mathrm{HF}$ e aqueceu-se até a secura. Garantiu-se que todo HF fosse evaporado com sucessivas adições de pequenas porções de HCL e secagem. Dissolveu-se o resíduo obtido com $30 \mathrm{~mL}$ de $\mathrm{HCl} 5 \%$ e diluiu-se para $100 \mathrm{~mL}$ em balão volumétrico.

\section{Procedimento}

\section{Determinação espectrofotométrica de ferro e titânio com} Tiron ou CSA

Uma vez que a composição média das amostras era conhecida, transferiu-se para um balão volumétrico de $25 \mathrm{~mL}$ uma alíquota da solução padrão ou da amostra contendo de 125,0 a $625,0 \mu \mathrm{g}$ de $\mathrm{Fe}$ ou Ti. Adicionou-se, então, 5,0 mL da solução de Tiron ou CSA, conforme o caso, e $10 \mathrm{~mL}$ da solução tampão $\mathrm{pH} 4,75$. Completou-se o volume com água desionizada, agitouse e mediu-se as absorvâncias dos complexos em 565 e $376 \mathrm{~nm}$ quando utilizou-se o Tiron ou em 500 e $360 \mathrm{~nm}$ quando usouse o CSA, utilizando os respectivos brancos dos reagentes. Montou-se o sistema de equações com as absorvâncias obtidas e calculou-se as concentrações dos metais.

\section{Determinação de ferro e titânio por ICP-AES ou FAAS}

Transferiu-se para um balão volumétrico de $25 \mathrm{~mL}$ uma alíquota da solução padrão ou de amostra contendo de 5,0 a $125,0 \mu \mathrm{g}$ de Fe e de 250,0 a $1250 \mu \mathrm{g}$ de Ti. Completou-se o volume com solução de ácido clorídrico 5\%. Para a determinação de titânio por FAAS foi necessário adicionar cálcio às soluções de referência utilizadas na curva.

\section{RESULTADOS E DISCUSSÃO}

\section{Determinação espectrofotométrica de ferro e titânio com Tiron}

Em pH 4,75, o Tiron apresentou absorção máxima em 218 $\mathrm{nm}$ e os complexos formados com ferro e titânio apresentaram absorção máxima em 565 e $376 \mathrm{~nm}$, respectivamente, como mostra a Figura 1. A determinação de ferro em $565 \mathrm{~nm}$ é isenta da interferência de titânio, entretanto para a determinação de titânio foi empregada a propriedade aditiva das absorvâncias, visto que o complexo ferro-Tiron absorve em $376 \mathrm{~nm}$. Tanto o reagente como os dois complexos metálicos mostraram-se estáveis por pelo menos $24 \mathrm{~h}$ em pH 4,75.

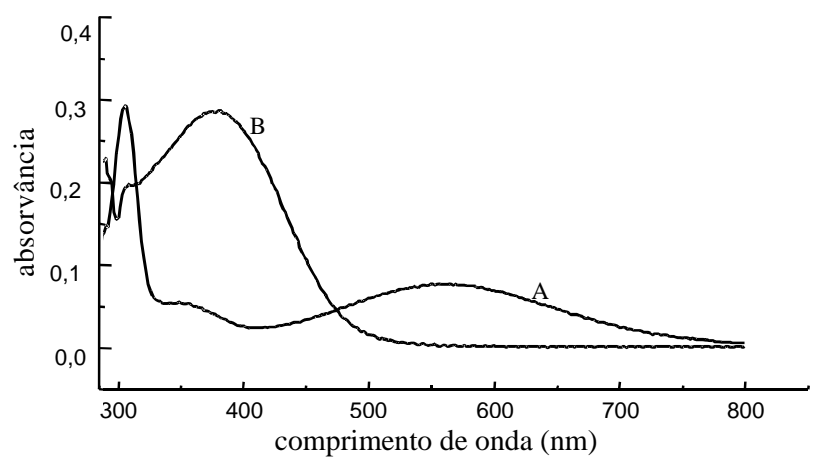

Figura 1. Espectros de absorção dos complexos Fe-Tiron e Ti-Tiron em pH 4,75: A) 3,58 $\times 10^{-5} \mathrm{~mol} \mathrm{~L}^{-1}$ de Fe; B) 4,18 $\times 10^{-5} \mathrm{~mol} \mathrm{~L}^{-1}$ de Ti.

Realizando o estudo do efeito do $\mathrm{pH}$, verificou-se que os complexos Fe-Tiron e Ti-Tiron apresentam absorção máxima em $\mathrm{pH}$ na faixa compreendida entre 4,4 a 5,0, e de 3,6 a 5,1 respectivamente. Escolheu-se, então, tampão ácido acético/acetato de sódio ajustado em $\mathrm{pH} 4,75$ para controle do $\mathrm{pH}$ das soluções.

O efeito da concentração do tampão acetato de sódio / ácido acético (pH 4,75) sobre os complexos do Tiron com o Fe e Ti foi estudado e os resultados demonstraram que a quantidade de acetato não modifica o sinal da absorvância de maneira significativa dentro da faixa de 0,15 a $0,40 \mathrm{~mol} \mathrm{~L}^{-1}$. Quanto à presença de Tiron no sistema, verificou-se que concentrações de reagente superiores a $5,0 \times 10^{-4} \mathrm{~mol} \mathrm{~L}^{-1}$ eram suficientes para garantir a completa complexação dos metais e consequentemente sinais de absorvância máximos e estáveis nas faixas de concentrações trabalhadas.

A influência da ordem de adição de reagentes sobre a formação dos complexos Ti-Tiron e Fe-Tiron foi estudada e verificouse que a formação do complexo com Fe não é afetada pela ordem 
em que os reagentes são adicionados. Entretanto, para o complexo do Ti-Tiron, observou-se variações na absorvância devido a ordem de adição. Verificou-se que maiores sinais de absorvância foram obtidos quando a solução tampão era adicionada após a adição do Tiron e do titânio. Sendo assim, adotou-se a ordem de adição Tiron + amostra + tampão para o procedimento.

Para avaliação da seletividade do Tiron, visando a aplicação do reagente na determinação do ferro e titânio em cimento, foram preparadas soluções contendo esses dois metais juntamente com os possíveis interferentes. Na composição do cimento Portland comum estão presentes normalmente o $\mathrm{Ca}, \mathrm{Sr}$, Al, P, Na, Mn, Mg, K, Li, Zr, Si. Para este estudo, simulouse a proporção média das espécies encontrada na literatura para cimento Portland comum ${ }^{12}$. Fixou-se o limite de tolerância de erro em $\pm 5 \%$ do sinal de absorvância obtido para soluções contendo $3,58 \times 10^{-5} \mathrm{~mol} \mathrm{~L}^{-1}$ de Fe e $1,04 \times 10^{-5} \mathrm{~mol} \mathrm{~L}^{-1} \mathrm{de}$ Ti. Conforme pode ser visto na Tabela 3 , dentro das faixas estudadas, não ocorreu interferência de nenhum dos elementos. Preparou-se também uma mistura sintética com todas as espécies simulando a proporção média do cimento Portland e não foi observada interferência.

As curvas analíticas foram preparadas segundo o procedimento experimental e verificou-se obediência à lei de Beer na faixa de concentração trabalhada: $8,95 \times 10^{-5} \mathrm{~mol} \mathrm{~L}^{-1}$ até 4,48 x $10^{-4} \mathrm{~mol} \mathrm{~L}^{-1}$ para o $\mathrm{Fe}$ e $\mathrm{Ti}$. As equações obtidas foram: $\mathrm{A}_{\mathrm{Fe}(565)}=4,24 \times 10^{3} \mathrm{C}_{\mathrm{Fe}}+0,002(\mathrm{R}=0,9999), \mathrm{A}_{\mathrm{Fe}(376) \mathrm{n}}=2,29$ $\mathrm{x} 10^{3} \mathrm{C}_{\mathrm{Fe}}+0,004(\mathrm{R}=0,9992)$ e $\mathrm{A}_{\mathrm{Ti}(376)}=1,48 \times 10^{4} \mathrm{C}_{\mathrm{Ti}}$ $(R=0,9999)$. A concentração de ferro foi obtida diretamente através da absorvância medida em $565 \mathrm{~nm}$. Para titânio a concentração foi encontrada baseando-se na propriedade aditiva da absorvância medida em $376 \mathrm{~nm}$ :

$$
\begin{aligned}
\mathrm{C}_{\mathrm{Fe}} & =\frac{\mathrm{A}_{565}-0,002}{4,24 \times 10^{3}} \\
\mathrm{C}_{\mathrm{Ti}} & =\frac{\mathrm{A}_{376}-\left(2,29 \times 10^{3} \mathrm{C}_{\mathrm{Fe}}+0,004\right)}{1,48 \times 10^{4}}
\end{aligned}
$$

Os limites de detecção $(3 \sigma)$ e os coeficientes de variação para determinação de Fe e Ti foram estimados em 5,37 x $10^{-7}$ e $4,18 \times 10^{-7} \mathrm{~mol} \mathrm{~L}^{-1}$ e $1,50 \%$ e $1,30 \%(n=15)$ respectivamente. Os limites de quantificação obtidos foram $3,25 \times 10^{-6} \mathrm{~mol} \mathrm{~L}^{-1}$ para o ferro e $1,04 \times 10^{-6} \mathrm{~mol} \mathrm{~L}^{-1}$ para o titânio.

\section{Determinação espectrofotométrica de ferro e titânio com CSA}

O CSA e os seus complexos formados com ferro e titânio apresentaram baixa solubilidade em água. O uso do surfactante Brij-35 possibilitou a solubização do reagente e dos complexos evitando o uso de solventes orgânicos. Na presença do Brij-35 e em meio tamponado em pH 4,75, o CSA apresentou absorção máxima em $310 \mathrm{~nm}$ enquanto seus complexos com o ferro e titânio apresentam comprimento de onda de absorção máxima em 500 e 360 nm, respectivamente, como mostra a Figura 2. O complexo titânio-CSA não apresenta absorção em $500 \mathrm{~nm}$, entretanto o complexo Fe-CSA absorve em $360 \mathrm{~nm}$ de modo que foi empregada a propriedade aditiva das absorvâncias para a determinação do titânio. Tanto o reagente como os dois complexos apresentaram ser estáveis por pelo menos 24 h em pH 4,75.

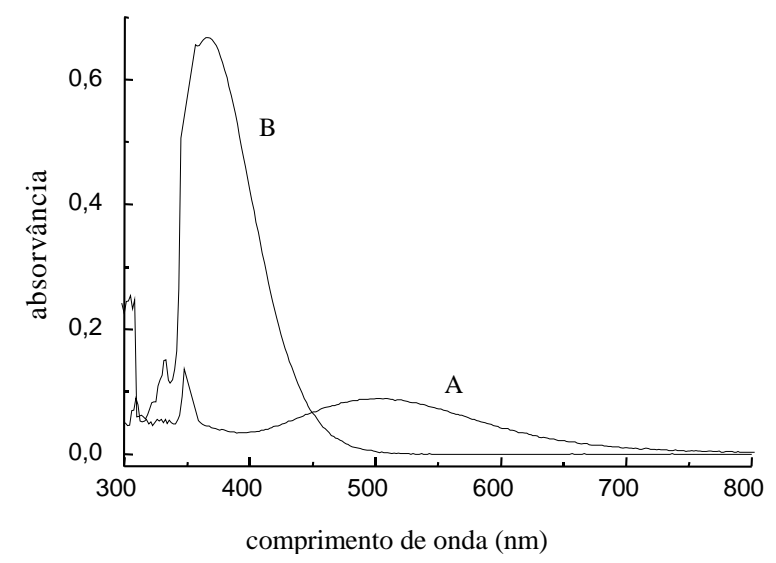

Figura 2. Espectros de absorção dos complexos Fe-CSA e Ti-CSA em pH 4,75: A) $3,58 \times 10^{-5} \mathrm{~mol} \mathrm{~L}^{-1}$ de $\mathrm{Fe}$; B) 4,18 $\times 10^{-5} \mathrm{~mol} \mathrm{~L}^{-1}$ de Ti.

$\mathrm{O}$ estudo do efeito do $\mathrm{pH}$ mostrou que melhores sinais de absorvância são obtidos em $\mathrm{pH}$ 4,5 a 5,5 para o complexo formado com o ferro e em pH 4,5 a 5,0 para o titânio. Escolheu-se tampão ácido acético/acetato de sódio ajustado em $\mathrm{pH} 4,75$ para controle do $\mathrm{pH}$ do meio. Verificou-se que a concentração de acetato no meio não influencia de maneira significativa o sinal de absorvância para concentrações entre 0,20 a $0,60 \mathrm{~mol} \mathrm{~L}^{-1}$.

Verificou-se a influência da quantidade de CSA adicionada sobre a absorvância dos complexos formados com o ferro e titânio. Observou-se que, para a faixa de concentração trabalhada, a quantidade mínima de reagente que deve estar presente no meio é de $0,58 \times 10^{-2} \mathrm{~mol} \mathrm{~L}^{-1}$.

No estudo da ordem de adição observou-se um comportamento semelhante ao obtido com o uso de Tiron: o complexo Fe-CSA não apresenta variações nos sinais de absorvância, para diferentes ordens de adição, enquanto que, para o complexo Ti-CSA, melhores sinais são obtidos quando a solução tampão é adicionada após o metal e o reagente. Portanto a ordem de adição sugerida é CSA + amostra + tampão.

Tabela 3. Estudo dos interferentes para os complexos do Fe e Ti com o Tiron*.

\begin{tabular}{cccc}
\hline Metal Adicionado & Concentração $\left(\mathrm{mol} \mathrm{L}^{-1}\right)$ & Abs. do Fe em $565 \mathrm{~nm}$ & Abs. do Ti em 376nm \\
\hline Nenhum & - & $0,144 \pm 0,001$ & $0,648 \pm 0,002$ \\
$\mathrm{Ca}$ & $8,98 \times 10^{-4}$ & $0,138 \pm 0,004$ & $0,668 \pm 0,004$ \\
$\mathrm{Sr}$ & $2,28 \times 10^{-6}$ & $0,142 \pm 0,004$ & $0,651 \pm 0,017$ \\
$\mathrm{Al}$ & $7,41 \times 10^{-5}$ & $0,140 \pm 0,003$ & $0,656 \pm 0,000$ \\
$\mathrm{P}$ & $1,29 \times 10^{-6}$ & $0,143 \pm 0,003$ & $0,667 \pm 0,005$ \\
$\mathrm{Na}$ & $3,48 \times 10^{-6}$ & $0,144 \pm 0,000$ & $0,663 \pm 0,002$ \\
$\mathrm{Mn}$ & $5,46 \times 10^{-5}$ & $0,141 \pm 0,001$ & $0,646 \pm 0,004$ \\
$\mathrm{Mg}$ & $1,65 \times 10^{-5}$ & $0,140 \pm 0,000$ & $0,638 \pm 0,001$ \\
$\mathrm{~K}$ & $1,53 \times 10^{-5}$ & $0,144 \pm 0,001$ & $0,645 \pm 0,008$ \\
$\mathrm{Li}$ & $2,88 \times 10^{-5}$ & $0,142 \pm 0,001$ & $0,650 \pm 0,003$ \\
$\mathrm{Zr}$ & $1,31 \times 10^{-5}$ & $0,635 \pm 0,004$ \\
\hline
\end{tabular}

* Testes realizados na presença de $3,58 \times 10^{-5} \mathrm{~mol} \mathrm{~L}^{-1}$ de Fe e $1,04 \times 10^{-5} \mathrm{~mol} \mathrm{~L}^{-1}$ de Ti seguindo o procedimento indicado na parte experimental. 
Para o estudo das interferências, utilizou-se soluções contendo $3,58 \times 10^{-1} \mathrm{~mol} \mathrm{~L}^{-1}$ de Fe e $1,04 \times 10^{-1} \mathrm{~mol} \mathrm{~L}^{-1}$ de Ti e simulou-se a composição média do cimento Portland comum ${ }^{12}$. Não foi encontrada interferência para nenhum dos íons metálicos testados, dentro do limite de tolerância de $\pm 5 \%$ do sinal do valor da absorvância (Tabela 4). Preparou-se também uma mistura sintética com todas as espécies simulando a proporção média do cimento Portland e não foi observada interferência.

As curvas analíticas foram preparadas segundo o procedimento experimental e verificou-se obediência à lei de Beer na faixa de concentração trabalhada: $3,58 \times 10^{-6}$ até $8,95 \times 10^{-5}$ mol L ${ }^{-1}$ para o $\mathrm{Fe}$ e $\mathrm{Ti}$. As equações obtidas foram: $\mathrm{A}_{\mathrm{Fe} 500}=$ $3,18 \times 10^{3} \mathrm{C}_{\mathrm{Fe}}-0,002(\mathrm{R}=0,9996), \mathrm{A}_{\mathrm{Fe} 360}=1,62 \times 10^{3} \mathrm{C}_{\mathrm{Fe}}+$ $0,002(\mathrm{R}=0,9992)$ e $\mathrm{A}_{\mathrm{Ti} 360}=1,60 \times 10^{4} \mathrm{C}_{\mathrm{Ti}}+0,022(\mathrm{R}=$ 0,9995). A concentração de ferro foi obtida diretamente através da absorvância medida em 500 nm. Para titânio a concentração foi encontrada baseando-se na propriedade aditiva da absorvância medida em $360 \mathrm{~nm}$ :

$$
\begin{aligned}
\mathrm{C}_{\mathrm{Fe}} & =\frac{\mathrm{A}_{500}+0,002}{3,18 \times 10^{3}} \\
\mathrm{C}_{\mathrm{Ti}} & =\frac{\mathrm{A}_{360}-\left(1,62 \times 10^{3} \mathrm{C}_{\mathrm{Fe}}+0,024\right)}{1,60 \times 10^{4}}
\end{aligned}
$$

Os limites de detecção $(3 \sigma)$ e os coeficientes de variação para determinação de Fe e Ti foram estimados em $1,34 \times 10^{-6} \mathrm{e}$ $4,18 \times 10^{-7} \mathrm{~mol} \mathrm{~L}^{-1}$ e $3,30 \%$ e $1,40 \%(n=15)$ respectivamente. Os limites de quantificação foram estimados em 5,39 x $10^{-6}$ mol L ${ }^{-1}$ para o ferro e $8,67 \times 10^{-7} \mathrm{~mol} \mathrm{~L}^{-1}$ para o titânio.

\section{Determinação de ferro e titânio empregando ICP-AES}

Uma série de testes foram realizados com o objetivo de otimizar as condições para a determinação de ferro e titânio empregando ICP-AES. Os parâmetros investigados foram: linhas de emissão, interferentes, faixa de trabalho, limite de detecção (LOD) e a concentração equivalente à radiação de fundo (BEC). Para o estudo das interferências, preparou-se soluções sintéticas contendo os possíveis interferentes conforme a composição média do cimento Portland.

Diversas linhas empregadas para a determinação de ferro e titânio podem ser encontradas na literatura ${ }^{13}$. Para $\mathrm{Fe}$ as principais linhas disponíveis são a 259,940; 239,562 e 238,204 nm e para Ti 336,121; 334,941 e 323,452 nm. A seleção da linha de emissão foi feita levando-se em conta dois fatores principais: a sensibilidade e a ausência de interferentes, levando em consideração a composição média do cimento Portland comum. Confirmou-se que para a determinação de ferro a linha mais sensível é em 259,9 nm e para a determinação de titânio é em
$334,9 \mathrm{~nm}$. Os testes de interferência foram realizados preparando-se soluções sintéticas contendo ferro, titânio e os possíveis interferentes. Nenhuma interferência foi observada nas duas linhas, de modo que foram utilizadas para a determinação dos dois metais.

As equações obtidas para as curvas analíticas foram: $\mathrm{I}=5,59 \times 10^{6} \mathrm{C}_{\mathrm{Ti}}+3,21(\mathrm{R}=0,9999) \mathrm{e} \mathrm{I}=3,29 \times 10^{6} \mathrm{C}_{\mathrm{Fe}}+$ $3,12(\mathrm{R}=0,9999)$, sendo a intensidade (I) expressa em Kcounts e a concentração em mol L $\mathrm{e}^{-1}$. O limite de detecção $(3 \sigma)$ para a determinação de ferro e o coeficiente de variação foram estimados em $4,96 \times 10^{-8} \mathrm{~mol} \mathrm{~L}^{-1}$ e $0,96 \%$ respectivamente. O BEC para a determinação de ferro foi estimado em $1,44 \times 10^{-6}$ mol L ${ }^{-1}$. Para a determinação de titânio estimou-se o coeficiente de variação em $0,79 \%$, o limite de detecção $(3 \sigma)$ em $2,40 \times 10^{-8} \mathrm{~mol} \mathrm{~L}^{-1}$ e o BEC em $8,57 \times 10^{-7} \mathrm{~mol} \mathrm{~L}^{-1}$.

\section{Determinação de ferro e titânio empregando FAAS}

Nas determinações empregando espectrometria de absorção atômica com chama, foram analisados diversos parâmetros com o objetivo de se obter maior sensibilidade, precisão e ausência de interferências.

Para o estudo das interferências na determinação de ferro e titânio com FAAS preparou-se soluções sintéticas contendo os possíveis interferentes conforme a composição média do cimento Portland. Nenhum dos metais testados causou interferência na determinação de ferro na linha mais sensível $(248,3 \mathrm{~nm})$.

Para a determinação do titânio, foram testadas várias linhas possíveis $(364,3,365,4$ e 399,0 nm). Observou-se uma baixa sensibilidade para a determinação do titânio, mesmo com a utilização de uma chama óxido nitroso/acetileno e da linha mais sensível. Foi necessário realizar a dissolução de uma massa maior das amostras de cimento (massa $\cong 1,0 \mathrm{~g}$ e volume final de $50 \mathrm{~mL}$ ) de forma a viabilizar a quantificação do titânio. Verificou-se que a única linha que apresentava sensibilidade suficiente, para as concentrações de titânio presente na amostra, era 364,3 nm. Entretanto, trabalhando-se nesta linha, foi encontrada uma interferência positiva devido ao alto teor de cálcio presente na matriz. Para a determinação de titânio foi necessário então, adicionar cálcio às soluções de referência utilizadas na curva analítica (concentração final de cálcio no balão igual a $9,98 \times 10^{-2} \mathrm{~mol} \mathrm{~L}^{-1}$ ), desta maneira evitou-se o efeito de matriz causado pelo alto teor deste metal no cimento.

As curvas analíticas encontradas para a determinação dos dois metais foram: $\mathrm{A}=3,52 \times 10^{3} \mathrm{C}_{\mathrm{Fe}}+0,004$ para curvas traçadas numa faixa de concentração de $3,58 \times 10^{-6}$ a $8,95 \times$ $10^{-5} \mathrm{~mol} \mathrm{~L}^{-1}$ e $\mathrm{A}=1,52 \times 10^{2} \mathrm{C}_{\mathrm{Ti}}+0,002$ para uma faixa de concentração de $2,09 \times 10^{-4}$ a $1,04 \times 10^{-3} \mathrm{~mol} \mathrm{~L}^{-1}$. O limite de detecção para a determinação de ferro e o coeficiente de variação $(n=15)$ foram estimados em $8,59 \times 10^{-7} \mathrm{~mol} \mathrm{~L}^{-1}$ e $3,44 \%$, respectivamente. Para a determinação de titânio estimou-se o

Tabela 4. Teste de interferentes para os complexos do Fe e Ti com o CSA*

\begin{tabular}{cccc}
\hline Interferente & Concentração $\left(\mathrm{mol} \mathrm{L}^{-1}\right)$ & Abs. do Fe em 500nm & Abs. do Ti em 360nm \\
\hline Nenhum & - & $0,194 \pm 0,024$ & $0,760 \pm 0,048$ \\
$\mathrm{Ca}$ & $8,98 \times 10^{-4}$ & $0,192 \pm 0,031$ & $0,771 \pm 0,012$ \\
$\mathrm{Sr}$ & $2,28 \times 10^{-6}$ & $0,195 \pm 0,020$ & $0,799 \pm 0,006$ \\
$\mathrm{Al}$ & $7,41 \times 10^{-5}$ & $0,200 \pm 0,035$ & $0,800 \pm 0,068$ \\
$\mathrm{P}$ & $1,29 \times 10^{-6}$ & $0,196 \pm 0,013$ & $0,775 \pm 0,004$ \\
$\mathrm{Na}$ & $3,48 \times 10^{-6}$ & $0,197 \pm 0,009$ & $0,774 \pm 0,006$ \\
$\mathrm{Mn}$ & $5,46 \times 10^{-5}$ & $0,194 \pm 0,011$ & $0,778 \pm 0,014$ \\
$\mathrm{Mg}$ & $1,65 \times 10^{-5}$ & $0,194 \pm 0,006$ & $0,773 \pm 0,003$ \\
$\mathrm{~K}$ & $1,53 \times 10^{-5}$ & $0,198 \pm 0,009$ & $0,767 \pm 0,011$ \\
$\mathrm{Li}$ & $2,88 \times 10^{-5}$ & $0,190 \pm 0,005$ & $0,770 \pm 0,021$ \\
$\mathrm{Zr}$ & $1,31 \times 10^{-5}$ & $0,198 \pm 0,015$ & $0,791 \pm 0,037$ \\
\hline
\end{tabular}

* Testes realizados na presença de $3,58 \times 10^{-5} \mathrm{~mol} \mathrm{~L}^{-1}$ de Fe e $1,04 \times 10^{-5} \mathrm{~mol} \mathrm{~L}^{-1}$ de Ti seguindo o procedimento indicado na parte experimental. 
Tabela 5. Comparação de métodos para a análise de Fe em cimento

\begin{tabular}{|c|c|c|c|c|}
\hline Cimento & $\begin{array}{c}\% \underset{\mathrm{Fe}}{\mathrm{Fe}_{2} \mathrm{O}_{3}} \\
\text { AAS }\end{array}$ & $\begin{array}{c}\% \underset{\mathrm{ICP}}{\mathrm{Fe}_{2} \mathrm{O}_{3}} \\
\text { ICP }\end{array}$ & $\begin{array}{c}\% \mathrm{Fe}_{2} \mathrm{O}_{3} \\
\text { Tiron }\end{array}$ & $\begin{array}{c}\% \mathrm{Fe}_{2} \mathrm{O}_{3} \\
\mathrm{CSA}\end{array}$ \\
\hline$B A$ & $3,12 \pm 0,27$ & $3,07 \pm 0,27$ & $3,12 \pm 0,28$ & $3,09 \pm 0,12$ \\
\hline Itaú & $2,12 \pm 0,02$ & $2,11 \pm 0,03$ & $2,11 \pm 0,04$ & $2,11 \pm 0,01$ \\
\hline Nassau & $2,49 \pm 0,05$ & $2,40 \pm 0,03$ & $2,42 \pm 0,02$ & $2,42 \pm 0,05$ \\
\hline Poty & $2,47 \pm 0,30$ & $2,49 \pm 0,08$ & $2,48 \pm 0,04$ & $2,48 \pm 0,08$ \\
\hline Aratu & $1,90 \pm 0,10$ & $1,85 \pm 0,04$ & $1,88 \pm 0,01$ & $1,89 \pm 0,05$ \\
\hline Composto com escória & $2,06 \pm 0,05$ & $2,09 \pm 0,05$ & $2,10 \pm 0,01$ & $2,09 \pm 0,17$ \\
\hline Alto forno & $1,46 \pm 0,05$ & $1,44 \pm 0,08$ & $1,44 \pm 0,08$ & $1,44 \pm 0,12$ \\
\hline
\end{tabular}

Tabela 6. Comparação de métodos para a análise de Ti em cimento

\begin{tabular}{|c|c|c|c|c|}
\hline Cimento & $\begin{array}{c}\% \mathrm{TiO}_{2} \\
\text { AAS }\end{array}$ & $\begin{array}{c}\% \mathrm{TiO}_{2} \\
\mathrm{ICP}\end{array}$ & $\begin{array}{c}\% \mathrm{TiO}_{2} \\
\text { Tiron }\end{array}$ & $\begin{array}{c}\% \mathrm{TiO}_{2} \\
\mathrm{CSA}\end{array}$ \\
\hline$B A$ & $0,23 \pm 0,02$ & $0,21 \pm 0,00$ & $0,22 \pm 0,01$ & $0,20 \pm 0,01$ \\
\hline Nassau & $0,29 \pm 0,03$ & $0,28 \pm 0,01$ & $0,29 \pm 0,01$ & $0,28 \pm 0,02$ \\
\hline Poty & $0,25 \pm 0,03$ & $0,24 \pm 0,00$ & $0,24 \pm 0,00$ & $0,22 \pm 0,02$ \\
\hline Aratu & $0,35 \pm 0,02$ & $0,34 \pm 0,01$ & $0,35 \pm 0,01$ & $0,39 \pm 0,01$ \\
\hline Composto com escória & $0,32 \pm 0,03$ & $0,31 \pm 0,01$ & $0,32 \pm 0,01$ & $0,30 \pm 0,02$ \\
\hline Alto forno & $0,41 \pm 0,02$ & $0,41 \pm 0,02$ & $0,41 \pm 0,00$ & $0,41 \pm 0,02$ \\
\hline
\end{tabular}

coeficiente de variação $(n=15)$ em $1,37 \%$ e o limite de detecção em $8,36 \times 10^{-6} \mathrm{~mol} \mathrm{~L}^{-1}$.

\begin{abstract}
Aplicação
Os métodos estudados foram aplicados para a determinação de ferro e titânio em 7 amostras de cimento Portland de diferentes marcas encontradas no mercado. Os resultados estão mostrados nas Tabelas 5 e 6 . Os resultados estão expressos como média de 3 replicatas para cada uma das sete amostras com os respectivos desvios para um nível de confiança de $95 \%$. Os resultados obtidos através das quatro técnicas foram comparados e avaliados através da aplicação do teste t-pareado, com nível de confiança de $95 \%$, e verificou-se que não há diferença significativa entre os resultados.
\end{abstract}

\section{CONCLUSÕES}

Os complexos de ferro e titânio formados tanto com o CSA quanto com o Tiron mostraram-se estáveis numa boa faixa de $\mathrm{pH}$ e os respectivos métodos espectrofotométricos apresentaram uma boa linearidade, reprodutibilidade e sensibilidade. O reagente CSA apresentou a desvantagem de ser pouco solúvel em água, entretanto a adição do surfactante Brij-35 permitiu o trabalho em solução aquosa sem a adição de maiores quantidades de solventes orgânicos. O método espectrofotométrico com Tiron apresentou maior sensibilidade para ferro enquanto CSA foi mais sensível para a determinação de titânio.

Em relação aos métodos de espectrometria atômica, constatou-se que a determinação de Fe tanto por AAS como por ICPAES é livre de interferências apresentando boa sensibilidade e reprodutibilidade. A determinação de titânio por ICP-AES também mostrou-se livre de interferências e com boa sensibilidade. Já a determinação de Ti por AAS além de apresentar baixa sensibilidade, observou-se também problema de interferência do $\mathrm{Ca}$, contornado com o uso de equivalência de matriz para a construção da curva analítica.

\section{AGRADECIMENTOS}

Os autores agradecem à CAPES, CNPq e FINEP pelo apoio financeiro.

\section{REFERÊNCIAS}

1. Garcia, I. L.; Navarro, E.; Viñas, P.; Córdoba, M. H.; Fresenius J. Anal. Chem. 1997, 357, 642.

2. Sedaira, H.; Idriss, K. A.; Abdel-Aziz, M. S.; Analyst 1996, 121, 1079.

3. Majundar, A. K.; Savariar, C. P.; Anal. Chim. Acta 1959, $21,53$.

4. Yoe, John H.; Armstrong, A. R.; Anal. Chem. 1947, 19,100

5. Piippanen, T.; Jaatinen, J.; Tummavuori, J.; Fresenius $J$. Anal. Chem. 1997, 357, 405.

6. Law, Oi-Wah; Lam, L.; Luk, Shiu-Fai; Talanta 1995, $42,1265$.

7. Delgado, L. C.; Manning, D. C.; Analyst 1967, 92, 553.

8. Choi, Ka-Keung; Lam, Lik; Luk, Shiu-Fai; Talanta 1994, $41,1$.

9. Bautista, M. A.; Sirvent, C. P.; Garcia, L. I.; Córdoba, H. M.; Fresenius J. Anal. Chem. 1994, 350, 359.

10. Floyd, M. A.; Fassel, V. A.; D'Silva, A. P.; Anal. Chem. 1980, 52, 2168.

11. Thompson, M.; Hamsei, M. H.; Analyst 1985, 110, 1413-1419.

12. Fariñas, J. C.; Ortega, P.; Analusis 1992, 20, 221.

13. Wang, E.; Liu, A.; Microchem. J. 1991, 43, 191. 